\title{
Correction to: Incorporating radiomics into clinical trials: expert consensus endorsed by the European Society of Radiology on considerations for data-driven compared to biologically driven quantitative biomarkers
}

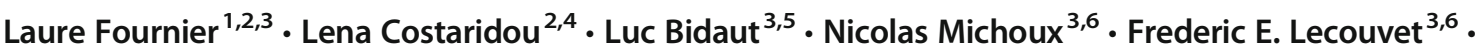 \\ Lioe-Fee de Geus-Oei ${ }^{3,7,8} \cdot$ Ronald Boellaard $^{2,9,10}$. Daniela E. Oprea-Lager ${ }^{3,9}$. Nancy A Obuchowski ${ }^{10,11}$. \\ Anna Caroli ${ }^{2,12}$ • Wolfgang G. Kunz ${ }^{3,13}$ • Edwin H. Oei ${ }^{2,14}$ • James P. B. O'Connor ${ }^{2,15}$ - Marius E. Mayerhoefer ${ }^{2,16}$. \\ Manuela Franca $^{2,17}$. Angel Alberich-Bayarri ${ }^{2,18}$. Christophe M. Deroose $3,19,20$. Christian Loewe . $^{2,21}$. \\ Rashindra Manniesing ${ }^{2,22}$. Caroline Caramella ${ }^{3,23}$. Egesta Lopci ${ }^{3,24}$ - Nathalie Lassau ${ }^{2,3,10,25}$ - Anders Persson ${ }^{2,26}$. \\ Rik Achten ${ }^{2,27} \cdot K$ Karen Rosendahl ${ }^{2,28}$. Olivier Clement ${ }^{1,2}$. Elmar Kotter ${ }^{2,29}$ - Xavier Golay ${ }^{2,10,30}$ - Marion Smits ${ }^{2,3,14}$. \\ Marc Dewey $^{2,31}$ - Daniel C. Sullivan ${ }^{2,10,32}$ - Aad van der Lugt ${ }^{2,14} \cdot$ Nandita M. deSouza ${ }^{2,3,10,33}$ (D) European Society of \\ Radiology 34
}

Published online: 10 March 2021

(C) The Author(s) 2021

\section{Correction to: European Radiology} https://doi.org/10.1007/s00330-020-07598-8

The original version of this article, published on 25 January 2021, unfortunately contained mistakes. The following corrections have therefore been made in the original: Firstly, "endorsed by the European Society of Radiology" was missing in the article title. Secondly, the institutional author "European Society of Radiology" was missing in the author line, including the related affiliation 34. Thirdly, the following sentence was missing in the Acknowledgements: This paper was endorsed by the ESR Executive Council in December 2020. The corrected title and author line are given above; the corrected affiliations are given below. The original article has been corrected.
Open Access This article is licensed under a Creative Commons Attribution 4.0 International License, which permits use, sharing, adaptation, distribution and reproduction in any medium or format, as long as you give appropriate credit to the original author(s) and the source, provide a link to the Creative Commons licence, and indicate if changes were made. The images or other third party material in this article are included in the article's Creative Commons licence, unless indicated otherwise in a credit line to the material. If material is not included in the article's Creative Commons licence and your intended use is not permitted by statutory regulation or exceeds the permitted use, you will need to obtain permission directly from the copyright holder. To view a copy of this licence, visit http://creativecommons.org/licenses/by/4.0/.

Publisher's note Springer Nature remains neutral with regard to jurisdictional claims in published maps and institutional affiliations.
The online version of the original article can be found at https://doi.org/ $10.1007 / \mathrm{s} 00330-020-07598-8$

Nandita M. deSouza

nandita.deSouza@icr.ac.uk

Extended author information available on the last page of the article 


\section{Affiliations}

Laure Fournier $^{1,2,3} \cdot$ Lena Costaridou $^{2,4} \cdot$ Luc Bidaut $^{3,5} \cdot$ Nicolas Michoux $^{3,6} \cdot$ Frederic E. Lecouvet $^{3,6}$. Lioe-Fee de Geus-Oei ${ }^{3,7,8} \cdot$ Ronald Boellaard ${ }^{2,9,10}$ • Daniela E. Oprea-Lager ${ }^{3,9} \cdot$ Nancy A Obuchowski $^{10,11}$. Anna Caroli ${ }^{2,12}$ - Wolfgang G. Kunz ${ }^{3,13} \cdot$ Edwin H. Oei ${ }^{2,14}$ - James P. B. O'Connor ${ }^{2,15}$ - Marius E. Mayerhoefer ${ }^{2,16}$. Manuela Franca $^{2,17}$. Angel Alberich-Bayarri ${ }^{2,18}$. Christophe M. Deroose ${ }^{3,19,20}$. Christian Loewe ${ }^{2,21}$. Rashindra Manniesing ${ }^{2,22}$. Caroline Caramella ${ }^{3,23}$ - Egesta Lopci ${ }^{3,24} \cdot$ Nathalie Lassau ${ }^{2,3,10,25}$. Anders Persson ${ }^{2,26}$. Rik Achten ${ }^{2,27}$ - Karen Rosendahl ${ }^{2,28}$. Olivier Clement ${ }^{1,2}$ - Elmar Kotter ${ }^{2,29} \cdot$ Xavier Golay $^{2,10,30}$ • Marion Smits ${ }^{2,3,14}$. Marc Dewey ${ }^{2,31}$ - Daniel C. Sullivan ${ }^{2,10,32} \cdot$ Aad van der Lugt ${ }^{2,14} \cdot$ Nandita M. deSouza $^{2,3,10,33}$ (D) European Society of Radiology

1 PARCC, INSERM, Radiology Department, AP-HP, Hopital europeen Georges Pompidou, Université de Paris, F-

75015 Paris, France

2 European Imaging Biomarkers Alliance (EIBALL), European Society of Radiology, Vienna, Austria

3 Imaging Group, European Organisation of Research and Treatment in Cancer (EORTC), Brussels, Belgium

4 School of Medicine, University of Patras, University Campus, Rio, 26500 Patras, Greece

5 College of Science, University of Lincoln, Lincoln LN6 7TS, UK

6 Department of Radiology, Institut de Recherche Expérimentale et Clinique (IREC), Cliniques Universitaires Saint Luc, Université Catholique de Louvain (UCLouvain), B-1200 Brussels, Belgium

7 Department of Radiology, Leiden University Medical Center, Leiden, The Netherlands

8 Biomedical Photonic Imaging Group, University of Twente, Enschede, The Netherlands

9 Department of Radiology \& Nuclear Medicine, Cancer Centre Amsterdam, Amsterdam University Medical Centers (VU University), Amsterdam, The Netherlands

10 Quantitative Imaging Biomarkers Alliance, Radiological Society of North America, Oak Brook, IL, USA

11 Department of Quantitative Health Sciences, Cleveland Clinic, Cleveland, OH, USA

12 Department of Biomedical Engineering, Istituto di Ricerche Farmacologiche Mario Negri IRCCS, Bergamo, Italy

13 Department of Radiology, University Hospital, LMU Munich, Munich, Germany

14 Department of Radiology \& Nuclear Medicine, Erasmus MC, University Medical Center, Rotterdam, The Netherlands

15 Division of Cancer Sciences, University of Manchester, Manchester, UK

16 Department of Biomedical Imaging and Image-guided Therapy, Medical University of Vienna, Vienna, Austria

17 Department of Radiology, CentroHospitalar Universitário do Porto, Instituto de Ciências Biomédicas de Abel Salazar, University of Porto, Porto, Portugal
18 Quantitative Imaging Biomarkers in Medicine (QUIBIM), Valencia, Spain

19 Nuclear Medicine, University Hospitals Leuven, Leuven, Belgium

20 Nuclear Medicine and Molecular Imaging, Department of Imaging and Pathology, KU Leuven, Leuven, Belgium

21 Division of Cardiovascular and Interventional Radiology, Dept. for Bioimaging and Image-Guided Therapy, Medical University of Vienna, Vienna, Austria

22 Department of Radiology and Nuclear Medicine, Radboud University Medical Center, 6525 GA Nijmegen, The Netherlands

23 Radiology Department, Hôpital Marie Lannelongue, Institut d'Oncologie Thoracique, Université Paris-Saclay, Le PlessisRobinson, France

24 Nuclear Medicine, Humanitas Clinical and Research Hospital IRCCS, Rozzano, MI, Italy

25 Imaging Department, Gustave Roussy Cancer Campus Grand, Paris, UMR 1281, INSERM, CNRS, CEA, Universite Paris-Saclay, Saint-Aubin, France

26 Department of Radiology, and Department of Health,Medicine and Caring Sciences, Center for Medical Image Science and Visualization (CMIV), Linköping University, Linköping, Sweden

27 Department of Radiology and Medical Imaging, Ghent University Hospital, Gent, Belgium

28 Department of Radiology, University Hospital of North Norway, Tromsø, Norway

29 Department of Radiology, University Medical Center Freiburg, Freiburg, Germany

30 Queen Square Institute of Neurology, University College London, London, UK

31 Department of Radiology, Charité Universitätsmedizin Berlin, Berlin, Germany

32 Dept. of Radiology, Duke University, 311 Research Dr, Durham, NC 27710, USA

33 Division of Radiotherapy and Imaging, The Institute of Cancer Research and Royal Marsden NHS Foundation Trust, London, UK

34 Am Gestade 1, 1010 Vienna, Austria 